\title{
In Vitro Fertilization and Cortical Granule Distribution of Bovine Oocytes Having Heterogeneous Ooplasm with Dark Clusters
}

\author{
Masashi NAGANO, Yoshiyuki TAKAHASHI and Seiji KATAGIRI \\ Laboratory of Theriogenology, Department of Veterinary Clinical Sciences, Graduate School of Veterinary Medicine, Hokkaido \\ University, Sapporo 060-0818, Japan
}

(Received 12 November 1998/Accepted 19 January 1999)

ABSTRACT. In vitro maturation, fertilization and subsequent development of oocytes with homogeneous (category 1), or heterogeneous
ooplasm (category 2) were investigated. No significant differences were observed in the nuclear maturation and total fertilization rates
between the two categories. However, category 2 oocytes showed a higher normal fertilization rate due to their lower incidence of
polyspermy as compared to category 1 oocytes. Electron microscopic study revealed that all category 2 oocytes had cortical granules
lined up next to the plasma membrane, and that some category 1 oocytes still had small clusters of cortical granules after maturation.
Although the proportion of cleaved zygotes was higher in category 2, the percentages of cleaved zygotes that developed to the blastocyst
stage did not differ between the two categories. These results demonstrate that oocytes with heterogeneous ooplasm have a higher
capacity for normal fertilization due to the reduction in polyspermy. This can be attributed to the normal distribution of cortical granules
in category 2 oocytes after maturation. - KEY woRDs: bovine, cortical granule, fertilization, morphology. J. Vet. Med. Sci. 61(5): 531-535, 1999

Bovine oocytes from small antral follicles exhibit a wide variety of morphological characteristics [8, 11]. It has been widely accepted that oocytes with homogeneous ooplasm and compact multilayered cumulus investment are suitable for in vitro maturation [11]. The number of oocytes with heterogeneous ooplasm having dark clusters collected from the ovary is more than double (approximately 25\%) that of the oocytes with homogeneous ooplasm [8]. Recent studies have suggested that the oocytes with heterogeneous ooplasm with dark clusters have a similar or higher capacity for in vitro maturation $[4,5]$, fertilization $[8,12]$ and development $[2,7,8]$ than the oocytes with homogeneous ooplasm. However, the previous results were inconsistent and fragmental, since in vitro maturation, fertilization, or subsequent development were separately examined by different groups under the different culture conditions. Moreover, the relationship between the developmental capacity and the ultrastructural features of the oocytes with various morphologies have not been examined.

In this study, therefore, we compared the in vitro maturation, fertilization and subsequent developmental competence of oocytes with homogeneous ooplasm and the oocytes having heterogeneous ooplasm with dark clusters. The relationship between developmental capacity and ultrastructural features was also evaluated using an electron microscope.

\section{MATERIALS AND METHODS}

Oocyte classification: The cumulus-oocyte complexes (COCs) were aspirated from small antral follicles of slaughterhouse-obtained bovine ovaries as described previously [15]. They were washed two times in Hepesbuffered Tyrode's medium (TALP-Hepes) [1] supplemented with $3 \mathrm{mg} / \mathrm{m} l$ bovine serum albumin (BSA, fraction $\mathrm{V}$,
Sigma Chemical Co., St. Louis, U.S.A.), $0.2 \mathrm{mM}$ sodium pyruvate (Sigma) and $50 \mu \mathrm{g} / \mathrm{m} l$ gentamicin sulfate (Sigma). During the washing procedure, oocytes were examined, selected and classified under a stereomicroscope by the following criteria. Category 1 represented oocytes with homogeneous ooplasm and multilayered cumulus investment. Category 2 represented oocytes having heterogeneous ooplasm with dark clusters, and multilayered cumulus investment.

In vitro maturation, fertilization and culture: In vitro maturation, fertilization and culture were carried out using the procedure described previously [15]. Briefly, the COCs were washed once in the maturation medium; $25 \mathrm{mM}$ Hepesbuffered TCM 199 supplemented with $10 \%$ fetal calf serum (Gibco Laboratories, Grand Island, NY, U.S.A.), 0.02 units/ $\mathrm{m} l$ follicle-stimulating hormone (Sigma), $1 \mu \mathrm{g} / \mathrm{m} l$ estradiol$17 \beta$ (Sigma) and $0.2 \mathrm{mM}$ sodium pyruvate and $50 \mu \mathrm{g} / \mathrm{m} l$ gentamicin sulfate. The oocytes in each category were cultured separately for $22 \mathrm{hr}$ in a $50 \mu \mathrm{l}$ droplet of maturation medium (10 or 11 oocytes per droplet) covered with paraffin oil at $39^{\circ} \mathrm{C}$ in a humidified atmosphere of $5 \% \mathrm{CO}_{2}$ in air. Frozen bovine semen collected from a Holstein bull was used for in vitro fertilization. After thawing the semen in a $37^{\circ} \mathrm{C}$ water bath for $30 \mathrm{sec}$, the motile sperm were separated using a discontinuous gradient with 45 and $90 \%$ Percoll (Pharmacia BioProcess, Uppsala, Sweden) by centrifugation at $700 \times g$ for $20 \mathrm{~min}$. The sperm were subsequently washed with a modified Brackett and Oliphant (mBO) medium [3] by centrifugation at $500 \times g$ for $5 \mathrm{~min}$. The mature oocytes were co-incubated with the sperm at the concentration of 5 $\times 10^{6} \mathrm{sperm} / \mathrm{m} l$ in a $100 \mu l$ droplet of $\mathrm{mBO}$ medium containing $3 \mathrm{mg} / \mathrm{m} l$ fatty-acid-free BSA (Sigma) and 2.5 $\mathrm{mM}$ theophylline (Sigma) covered with paraffin oil for 20 $\mathrm{hr}$ at $39^{\circ} \mathrm{C}$ in a humidified atmosphere of $5 \% \mathrm{CO}_{2}$ in air. After insemination, presumptive zygotes were stripped of 
cumulus cells by vortexing in TALP-Hepes. They were washed three times with culture medium, a modified synthetic oviduct fluid supplemented with 20 amino acids and insulin (mSOFai) [15]. Groups of 25 to 35 presumptive zygotes were cultured in $50 \mu \mathrm{l}$ droplets of mSOFai covered with paraffin oil at $39^{\circ} \mathrm{C}$ in a humidified atmosphere of $5 \%$ $\mathrm{CO}_{2}, 5 \% \mathrm{O}_{2}$ and $90 \% \mathrm{~N}_{2}$.

Experiment 1: The oocytes in each category were cultured separately for 12 or $22 \mathrm{hr}$ in the maturation medium to clarify the difference in nuclear maturation between the two categories. At the end of culture, the COCs were vortexed and stripped of cumulus investment. The naked oocytes were fixed with ethanol:acetic acid (3:1), and stained with $1 \%$ aceto-orcein. Their nuclear status was examined under a phase-contrast microscope.

Experiment 2: The fertilization rate of oocytes in each category was evaluated at $20 \mathrm{hr}$ post-insemination (hpi), after they were fixed and stained as described above. Oocytes were considered to be fertilized when they had an enlarged sperm head(s) or male pronucleus(ei) with the corresponding sperm tail(s). The normal fertilization was determined by the presence of a pair of pronuclei and a corresponding sperm tail.

Experiment 3: This experiment was conducted to evaluate the subsequent developmental capacity of oocytes in each category. After in vitro maturation and in vitro fertilization, presumptive zygotes derived from the oocytes in each category were first cultured for $52 \mathrm{hr}$ in mSOFai. At 72 hpi, they were divided into 5 groups according to their developmental stages (1-cell, 2-cell, 3- to 4-cell, 5- to 7-cell and $\geq 8$-cell stages). The embryos in each group were then separately cultured in fresh culture medium until 168-170 hpi to determine their development to the blastocyst stage. All of the blastocysts obtained were fixed and their total cell numbers were counted using the air-drying method described elsewhere [14].

Experiment 4: This experiment evaluated the difference in the ultrastructural features, especially cortical granule distribution, between the two categories of oocytes, by using a transmission electron microscope. Before and after 22-hr culture for maturation, the oocytes in each category were fixed in $2.5 \%$ glutaraldehyde in $0.1 \mathrm{M}$ phosphate-buffered saline at $\mathrm{pH} 7.3$, and post-fixed in $1.0 \%$ osmium tetroxide in the same buffer. They were then dehydrated in a graded series of ethanol, treated with propylene oxide, embedded in epoxy resin and sectioned. The semi-thin sections stained with $0.5 \%$ toluidine blue at $\mathrm{pH} 7.5$ were observed under a light microscope. The ultra-thin sections stained with ethanolic uranyl acetate and lead citrate were examined under a transmission electron microscope (JEM-1210; JEOL, Tokyo, Japan). The evaluations of ultrastructural features in the semi-thin and ultra-thin sections were performed on sections of the equatorial plane of the oocyte.

Statistical analysis: The experimental data on maturation, fertilization and total cell number in blastocysts were analyzed by Student's $t$-test. The data on in vitro development were analyzed by the chi-square test.

\section{RESULTS}

Experiment 1: There was no significant difference in the nuclear status between the two categories at 12 and $22 \mathrm{hr}$ after in vitro culture for maturation (Table 1).

Experiment 2: Although the total fertilization rates did not differ between the two categories, the normal fertilization rate in category 2 was higher than that of category $1(\mathrm{P}<0.05$, Table 2$)$. The incidence of polyspermy in category 1 was significantly higher than that of category $2(\mathrm{P}<0.01)$.

Experiment 3: The proportion of cleaved zygotes was significantly higher in category 2 than in category 1 at 72 hpi $(\mathrm{P}<0.01$, Table 3$)$. However, there were no significant differences between the two categories in the proportion of embryos at each developmental stage (2-cell, 3- to 4-cell, 5to 7 -cell or $\geq 8$-cell stage) based on the number of cleaved zygotes. The percentages of embryos that developed to the blastocyst stage and total cell numbers in the blastocysts were not different between the two categories.

Experiment 4: Before the culture for maturation, cortical granules were organized in large clusters (Fig. 1a) in all category 1 oocytes $(n=10)$ and in 5 of 10 category 2 oocytes. The rest of the category 2 oocytes $(n=5)$ presented cortical granules partly lined up next to the plasma membrane (Fig. 1b). The lipid droplets were more abundant and often larger in diameter in the category 2 oocytes (Fig. 1c).

After 22-hr culture for maturation, 7 of 15 category 2 oocytes had cortical granules located individually next to the plasma membrane. In the other category 2 oocytes $(n=8)$, cortical granules were organized in small clusters and partly lined up next to the plasma membrane. In category 1, large clusters of cortical granules were still observed in 7 of 15 oocytes. Four category 1 oocytes had cortical granules located individually next to the plasma membrane. In the rest of the category 1 oocytes $(n=4)$, cortical granules were organized in small clusters and partly

Table 1. Nuclear status of oocytes at $12 \mathrm{hr}$ - and $22 \mathrm{hr}$-culture for maturation in two categories

\begin{tabular}{lcc}
\hline & \multicolumn{2}{c}{ Oocyte categorya) } \\
\cline { 2 - 3 } Item & 1 & 2 \\
\hline 12 hr after culture & & \\
$\quad$ No. of oocytes cultured & 70 & 63 \\
$\quad$ No. of replicates) & $(3)$ & $(3)$ \\
$\%$ of metaphase I & $98.5 \pm 2.6$ & $98.3 \pm 2.9$ \\
\% of anaphase I & $1.5 \pm 2.6$ & $1.7 \pm 2.9$ \\
and telophase I & & \\
\hline 22 hr after culture & & \\
No. of oocytes cultured & 67 & 71 \\
(No. of replicates) & $(3)$ & $(3)$ \\
$\%$ of metaphase I & $4.7 \pm 4.6$ & $1.4 \pm 2.5$ \\
\% of metaphase II & $95.3 \pm 4.6$ & $98.7 \pm 2.5$ \\
\hline
\end{tabular}

Values are means \pm SD.

a) Category 1: oocytes with homogeneous ooplasm, Category 2: oocytes having heterogeneous ooplasm with dark clusters. 
lined up next to the plasma membrane.

\section{DISCUSSION}

The present results agreed with previous findings [4, 5] that oocytes having heterogeneous ooplasm with dark clusters have potential for nuclear maturation in vitro comparable to that of oocytes with homogeneous ooplasm. However, the maturation rate of oocytes with heterogeneous ooplasm after 24-hr culture was reported to be significantly higher than that of oocytes with homogeneous ooplasm [12]. The discrepancy may be due to the low maturation rate (approximately $80 \%$ ) after 24-hr culture of the oocytes with homogeneous ooplasm under $2 \% \mathrm{CO}_{2}$ in air in the previous report [12].

The normal fertilization and cleavage rate were higher in category 2 oocytes than in category 1 due to the low

Table 2. In vitro fertilization in two categories at $20 \mathrm{hr}$ postinsemination

\begin{tabular}{lcc}
\hline \multirow{2}{*}{ Item } & \multicolumn{2}{c}{ Oocyte category ${ }^{\mathrm{a})}$} \\
\cline { 2 - 3 } & 1 & 2 \\
\hline No. of oocytes inseminated & 134 & 131 \\
(No. of replicates) & $(5)$ & $(5)$ \\
$\%$ of total fertilization & $94.5 \pm 3.4$ & $96.0 \pm 4.1$ \\
\% of normal fertilization & $86.2 \pm 4.9^{\mathrm{b})}$ & $93.8 \pm 2.2^{\mathrm{c})}$ \\
\% of polyspermy & $8.3 \pm 3.4^{\mathrm{d})}$ & $2.2 \pm 2.0^{\mathrm{e})}$ \\
\hline
\end{tabular}

Values are means \pm SD.

a) see the footnote in Table 1 .

$\mathrm{b}, \mathrm{c})$ and d, e) Values with different superscripts differ significantly $(\mathrm{P}<0.05$ and $\mathrm{P}<0.01$, respectively). incidence of polyspermy. In previous reports [8, 12], however, there was no significant difference in the proportions of normally fertilized oocytes between the two groups that were comparable to the present categories 1 and 2. The disagreement with our results may be due to the difference in the experimental design used for in vitro fertilization; co-culturing with somatic cells [8] or using calcium ionophore A23185 and several concentrations of sperm for insemination [12]. In the present study, the total fertilization rates were constant at about $95 \%$ among replicates in both categories, whereas the fertilization rate varied between 30 and $90 \%$ in both categories in the previous study [12].

Cortical granules are distributed throughout the entire inner surface of the sphere of the oocyte during final maturation $[10,17]$. Exocytosis of cortical granules is the most common mechanism for the prevention of polyspermy. The clustering of the cortical granules after maturation is likely to be responsible for the frequently encountered abnormal cortical granule release, which may dispose the oocytes to polyspermic penetration [9]. In all category 2 oocytes, cortical granules lined up next to the plasma membrane after the culture for maturation, whereas some category 1 oocytes had small clusters of cortical granules. It is probable that category 1 oocytes could not acquire the ability to block polyspermy due to the improper distribution next to the plasma membrane.

It was shown that the difference in the cortical granule distribution was present in the oocytes with various morphologies before in vitro maturation [5], and was no longer observed after in vitro maturation [4]. However, the difference in the cortical granule distribution in each

Table 3. Developmental stages at $72 \mathrm{hr}$ post-insemination and development to blastocyst stage at $168-170 \mathrm{hr}$ post-insemination derived from oocytes of each category

\begin{tabular}{lcc}
\hline \multirow{2}{*}{ Item } & \multicolumn{2}{c}{ Oocyte category $^{\mathrm{a})}$} \\
\cline { 2 - 3 } & 1 & 2 \\
\hline No. of zygotes cultured & 254 & 256 \\
No. (\%) of cleaved zygotes ${ }^{\mathrm{b})}$ & $207(81.9)^{\mathrm{c})}$ & $235(91.6)^{\mathrm{d})}$ \\
No. (\%) of each cell stage/cleaved at 72 hpi & $43(20.8)$ & $36(15.3)$ \\
2-cell stage & $43(20.8)$ & $44(18.7)$ \\
3-4-cell stage & $40(19.3)$ & $45(19.1)$ \\
5-7-cell stage & $81(39.1)$ & $110(46.8)$ \\
$\geq 8$-cell stage & $42(20.3)$ & $55(23.4)$ \\
\hline Total no. (\%) of blastocysts/cleaved zygotes & $0(0.0)$ & 0 \\
No. (\%) of blastocysts derived from embryos at each stage at 72 hpi & \\
2-cell stage & $1(2.3)$ & $1(2.3)$ \\
3-4-cell stage & $7(17.5)$ & $9(20.0)$ \\
5-7-cell stage & $34(42.0)$ & $45(40.9)$ \\
$\geq 8$-cell stage & $129.5 \pm 48.6$ & $149.1 \pm 57.9$ \\
\hline Total cell no. in blastocysts (mean \pm SD) & & \\
\hline
\end{tabular}

Data were pooled for 6 replicates.

a) see the footnote in Table 1 .

b) The embryos that developed to $\geq 2$-cell stage were considered to be cleaved zygotes.

c, d) Values with different superscripts differ significantly $(\mathrm{P}<0.01)$. 


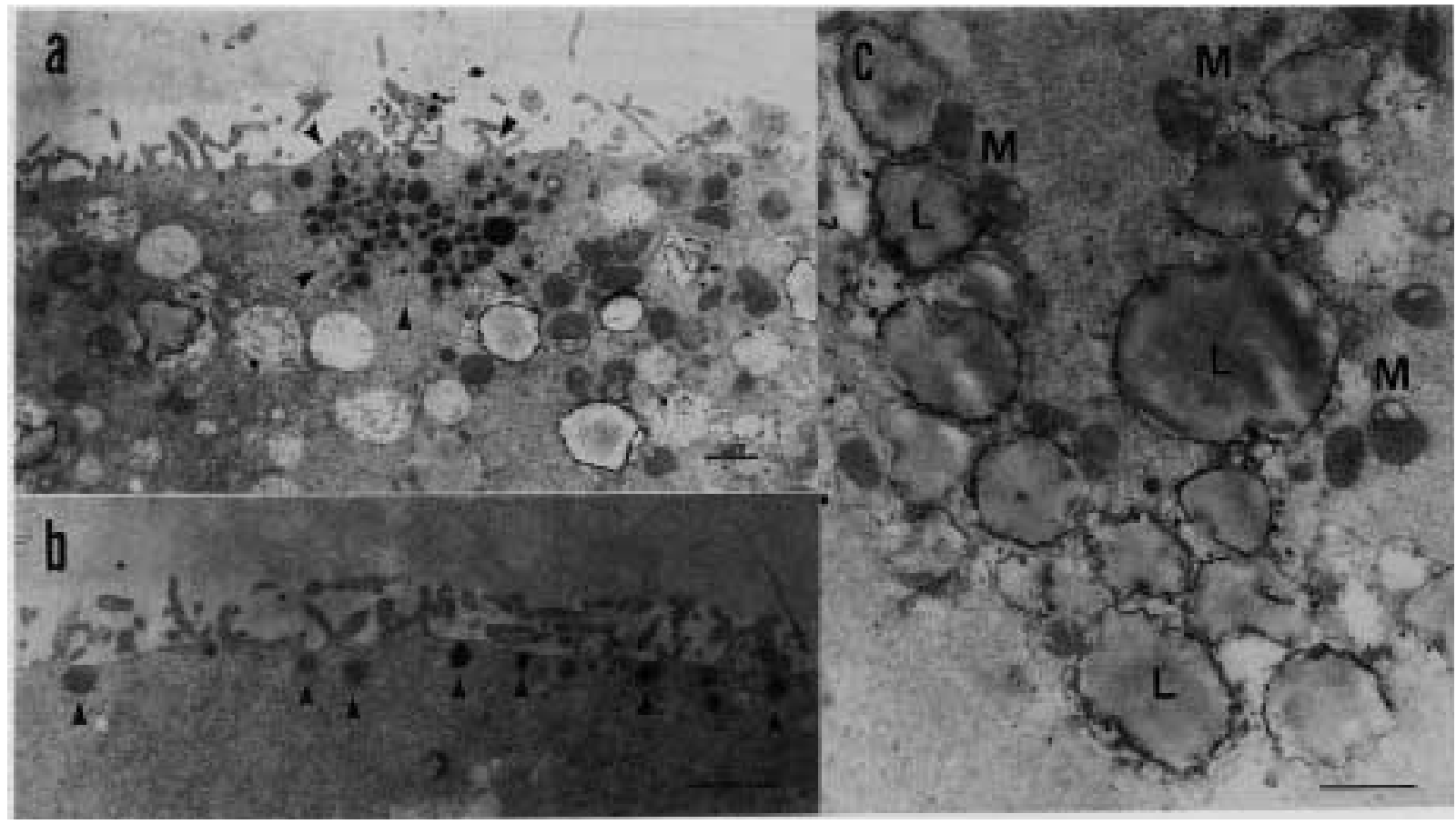

Fig. 1. Ultrastructural features of bovine oocytes under a transmission electron microscope. a; Cortical granules are organized in a large cluster (arrowhead). b; Cortical granules lined up next to the plasma membrane (arrowhead). c; The lipid droplets (L) are abundant and appear to cluster with mitochondria (M). Bar $=1 \mu \mathrm{m}$

category remained after maturation in the present study. One possible reason for this inconsistency might be the difference in the in vitro maturation system. Oocytes were previously co-cultured with granulosa cells in a large well without an oil covering [4].

In spite of the significant difference in the percentage of cleaved zygotes, the proportion of cleaved zygotes that developed to the blastocyst stage did not differ between the two categories. This finding agrees with previous reports $[7,8]$, and suggests that the developmental capacity of normally fertilized oocytes to the blastocyst stage does not differ between the two categories. However, the developmental rate of cleaved zygotes to the blastocyst stage in this study was lower than that obtained under our routine procedure (30-40\%) [16]. The present culture conditions may be suboptimal, since we divided embryos into several groups according to their developmental stages at $72 \mathrm{hpi}$. This procedure reduces the embryo density in culture and decreases the embryonic developmental rate $[2,6,13]$. Therefore, we might have failed to detect differences in the developmental rate of the two categories. Thus, we need to conduct further studies under optimal conditions.

It is concluded that oocytes with heterogeneous ooplasm have a higher capacity for normal fertilization than oocytes with homogeneous ooplasm, probably due to the reduction in polyspermy. This can be attributed to the normal distribution of cortical granules in the oocytes with heterogeneous ooplasm.
ACKNOWLEDGMENTS. This study was supported by a Grant-in-Aid for Scientific Research (No. 09660296) from the Ministry of Education, Science, Sports and Culture, Japan to Y. Takahashi. We thank the staff of the Hokkaido Livestock Improvement Association for the donation of frozen bull sperm and the Ebetsu Meat Inspection Office for the collection of bovine ovaries.

\section{REFERENCES}

1. Bavister, B. D., Leibfried, M. L. and Lieberman, G. 1983. Development of preimplantation embryos of the golden hamster in a defined culture medium. Biol. Reprod. 28: 235-247.

2. Blondin, P. and Sirard, M. A. 1995. Oocyte and follicular morphology as determining characteristics for developmental competence in bovine oocytes. Mol. Reprod. Dev. 41: 54-62.

3. Brackett, B. G. and Oliphant, G. 1975. Capacitation of rabbit spermatozoa in vitro. Biol. Reprod. 12: 260-274.

4. De Loos, F., Van Maurik, P., Van Beneden, T. and Kruip, T. A. M. 1992. Structural aspects of bovine oocyte maturation in vitro. Mol. Reprod. Dev. 31: 208-214.

5. De Loos, F., Van Vliet, C., Van Maurik, P. and Kruip, T. A. M. 1989. Morphology of immature bovine oocytes. Gamete Res. 24: 197-204.

6. Ferry, L., Mermillod, P., Massip, A. and Dessy, F. 1994. Bovine embryos cultured in serum-poor oviduct-conditioned medium need cooperation to reach the blastocyst stage. Theriogenology 42: 445-453.

7. Hawk, H. W. and Wall, R. J. 1994. Improved yields of bovine blastocysts from in vitro-produced oocytes. I. Selection 
of oocytes and zygotes. Theriogenology 41: 1571-1583.

8. Hazeleger, N. L., Hill, D. J., Stubbings, R. B. and Walton, J. S. 1995. Relationship of morphology and follicular fluid environment of bovine oocytes to their developmental potential in vitro. Theriogenology 43: 509-522.

9. Hyttel, P., Xu, K. P. and Greve, T. 1988. Ultrastructural abnormalities of in vitro fertilization of in vitro matured bovine oocytes. Anat. Embryol. 178: 47-52.

10. Kruip, T. A. M., Cran, D. G., Van Beneden, T. H. and Dieleman, S. J. 1983. Structural changes in bovine oocytes during final maturation in vivo. Gamete Res. 8: 29-47.

11. Leibfried, L. and First, N. L. 1979. Characterization of bovine follicular oocytes and their ability to mature in vitro. $J$. Anim. Sci. 48: 76-86.

12. Momozawa, K. and Fukuda, Y. 1995. In vitro maturation and in vitro fertilization of bovine oocytes with heterogeneous ooplasm. Anim. Sci. Technol. 66: 605-609 (In Japanese).

13. Pinyopummin, A., Takahashi, Y., Hishinuma, M. and
Kanagawa, H. 1994. In vitro development of mouse parthenogenetic embryos to blastocysts: Effect of embryo density. J. Reprod. Dev. 40: 55-58.

14. Takahashi, Y. and First, N. L. 1992. In vitro development of bovine one-cell embryos: influence of glucose, lactate, pyruvate, amino acids and vitamins. Theriogenology 37: 963-978.

15. Takahashi, Y., Hishinuma, M., Matsui, M., Tanaka, H. and Kanagawa, H. 1996. Development of in vitro matured/fertilized bovine embryos in a chemically defined medium: influence of oxygen concentration in the gas atmosphere. $J$. Vet. Med. Sci. 58: 897-902.

16. Takahashi, Y. and Kanagawa, H. 1998. Effects of glutamine, glycine and taurine on the development of in vitro fertilized bovine zygotes in a chemically defined medium. J. Vet. Med. Sci. 60: 433-437.

17. Thibault, D., Szöllösi, D. and Gérard, M. 1987. Mammalian oocyte maturation. Reprod. Nutr. Dev. 27: 865-896. 